\title{
Poloidal Field Design and Plasma Scenarios for FIRE
}

\author{
C. E. Kessel ${ }^{1}$ and R. H. Bulmer ${ }^{2}$ \\ ${ }^{1}$ Princeton Plasma Physics Laboratory, P.O. Box 451, Princeton, NJ 08543 \\ ${ }^{2}$ Lawrence Livermore National Laboratory, P.O. Box 808, Livermore, CA 94550
}

\begin{abstract}
The FIRE (Fusion Ignition Research Experiment) device is a compact copper magnet experiment to explore driven DT burning plasma operation. As part of the design study, the poloidal field requirements, self-consistent dynamic discharge evolutions, and the plasma vertical stability and control are examined. Reported here are the PF (poloidal field) coil locations and currents, and a full discharge simulation of the reference configuration. In addition, other configurations are briefly described, and vertical instability growth times and feedback control currents and voltages are given.
\end{abstract}

\section{INTRODUCTION}

The major plasma parameters for FIRE are $\mathrm{I}_{p}=6.44 \mathrm{MA}, \mathrm{R}=2.00 \mathrm{~m}, \mathrm{a}=0.525 \mathrm{~m}, \kappa_{x}=2.0$, $\delta_{x}=0.7$ and $\mathrm{B}_{T}=10.0 \mathrm{~T}$. The plasma, structure, and poloidal field coil model used in the analysis is shown in Fig. 1. Analysis was done to determine PF (poloidal field) coil locations and currents to provide plasma equilibria throughout the inductive discharge; for the reference $10.0 \mathrm{~T}$ and $6.44 \mathrm{MA}$, the high field 12.0 T and 7.7 MA, and long pulse DD 4.0 T and 2.0 MA configurations. The coil currents were calculated for varying li, $\beta_{p}$, and $\psi_{e x t}$ at each of the fiducial states. PF coil currents in combination with prescribed plasma density evolution were used in the Tokamak Simulation Code (TSC)[1] to determine plasma current profile and boundary evolution, poloidal flux consumption, and plasma transport behavior (temperature, bootstrap current, radiation, L-H transition, etc.). Vertical stability was analyzed to design passive stabilizing structure and vertical control simulations were done to examine the feedback control requirements. The flattop pulse lengths in these studies are the maximums determined by the magnets, while nuclear heating of structures may lead to shorter pulse lengths.

\section{PF COIL LOCATIONS AND CURRENTS}

Shown in Fig. 1 is the plasma, axisymmetric structure model, and PF coils. The PF coils are all located outside the TF (toroidal field) coil. The $3 \mathrm{PF}$ coils located in the center stack are referred to CS1-3, while the remaining PF coils are referred to as PF1-4. The coil locations are given in Table 1 , and were the result of several trade-offs with device radial build, TF coil structure, equilibrium and flux requirements, and various interferences. In addition to the coils locations, Table 1 gives the ohmic distribution that provides for a constant flux over the plasma region. This is used to advance the flux state without disturbing the plasma equilibrium.

The PF coil currents are given in Table 2, for the reference $21 \mathrm{~s}$ flattop discharge, at a series of fiducial states; initial magnetization (IM), start of discharge (SOD), start of flattop (SOF), start of burn (SOB), end of burn (EOB), end of cooling (EOC), and end of discharge (EOD). The nominal plasma parameters are also given. These resulting currents were determined by considering plasma equilibrium and flux consumption from TSC discharge simulations, coil heating and stress analysis, and power supply analysis. At the reference parameters (10.0 T and $6.44 \mathrm{MA})$ the design has sufficient margin in coil allowables to accomodate uncertainties in plasma parameter evolutions, particularly variations in $l_{i}$ and $\psi_{e x t}$. This is shown in Table 2, where the maximum current in each coil is given for variations of $l_{i}$ between 0.75 and 1.0 , and $\psi_{e x t}$ from -5.0 to +5.0 webers about the nominal value. PF coil currents were similarly developed for the a high field case (12.0 T and $7.7 \mathrm{MA}$ ) with a flattop of $12 \mathrm{~s}$, and a long pulse DD case $(4.0 \mathrm{~T}$ and $2.0 \mathrm{MA}$ ) with a flattop of $250 \mathrm{~s}$. These were also found to remain within coil heating, stress, and power supply allowables. 


\section{DYNAMIC PLASMA DISCHARGE SIMULATIONS}

TSC was used to develop full discharge simulations including plasma current rampup, heating to burn, flattop burn, burn termination, and plasma current rampdown. TSC is a two-dimensional time dependent free-boundary simulation code that advances the MHD equations describing the transport time-scale evolution of an axisymmetric magnetized tokamak plasma. For the present simulations the plasma density is prescribed by

$$
n(\psi, t)=n_{o}(t)\left[\left(1-\hat{\psi}^{2.0}\right)^{0.5}+0.3\right]
$$

leading to a peak to average density of 1.3, intended to represent an ELMing H-mode. The simulation includes neoclassical resistivity, bootstrap current, a time-averaged sawtooth model, a modified CoppiTang energy transport model, radiation, and alpha and fusion effects. Passive structures are included to obtain accurate estimates of flux consumption and response of feedback control systems. The passive structure for FIRE is a double walled vacuum vessel and copper passive stabilizer plates (both inboard and outboard). Since TSC must begin its simulation with a plasma present, a constant voltage is applied as an initial condition over the computational grid. This causes finite structure currents to exist at the beginning of the simulation, as would be expected after the plasma breakdown. Feedback systems for the plasma current and radial position were used (simulations were up-down symmetric), with all the PF coils contributing to current control, and PF4 providing radial position control.

Several plasma characteristics are assumed; the impurity is $3 \%$ beryllium, the effective particle confinement time is 5 times the energy confinement time, the energy confinement time is about $0.5 \mathrm{~s}$, the Harris[2] bootstrap model is used, $100 \%$ of the ICRF heating goes into ions, and the plasma edge temperature is $500 \mathrm{eV}$. The vacuum toroidal field is ramped up over $21 \mathrm{~s}$ to its flattop value of $10.0 \mathrm{~T}$ (reaching this value at SOF), and begins dropping at the end of the flattop (EOB) with its $\mathrm{L} / \mathrm{R}$ time scale (about $20 \mathrm{~s}$ ).

Plasma current rampup extends from $0(\mathrm{SOD})$ to $6(\mathrm{SOF}) \mathrm{s}$. The plasma starts as a circular $100 \mathrm{kA}$ plasma limited on the inboard wall, and is grown over the rampup to full size and shape. The plasma current is ramped up linearly from $100 \mathrm{kA}$ to $6.44 \mathrm{MA}$ in $6.0 \mathrm{~s}$. The plasma is diverted at about 3.2 $\mathrm{s}$, and the full $30 \mathrm{MW}$ of ICRF heating is applied at $4.8 \mathrm{~s}(\mathrm{Ip}=5.5 \mathrm{MA})$ causing the plasma to enter the H-mode. The heating during rampup was found to provide a robust entry into H-mode that could be maintained during the fast density rise that occurs later. The plasma consumes $31.3 \mathrm{~V}$-s giving a flux state of $11.2 \mathrm{~Wb}$ at SOF. The plasma startup trajectory remains in the stable region of the $l_{i}-q_{95}$ diagram. However, $q_{95}$ briefly drops below 3.0 at SOF. The Ejima coefficient is 0.35 , due to the heating during current rampup. The sawtooth radius reaches about 0.17 , which is slowed down by the heating. At the end of the plasma current rampup phase the peak electron density is $1.75 \times 10^{20} / \mathrm{m}^{3}$, the peak electron temperature is $17 \mathrm{keV}$ and ion temperature is $24 \mathrm{keV}, \beta_{N}$ reaches $0.75, P_{\alpha}$ is $10 \mathrm{MW}$, and $P_{\text {aux }}$ is $30 \mathrm{MW}$.

The approach to burn and flattop burn extend from 6 to 27 (EOB) s. Since heating begins in the plasma current rampup phase, there is no well defined heating to burn phase. From 6 to $8 \mathrm{~s}$, the peak electron density is increased to $4 \times 10^{20} / \mathrm{m}^{3}$. The density is further ramped more slowly to $5 \times 10^{20} / \mathrm{m}^{3}$ from 8 to $14 \mathrm{~s}$, to control the fusion power overshoot. At $7.5 \mathrm{~s}$ the heating power is dropped to $22 \mathrm{MW}$, and the alpha power rises to $50 \mathrm{MW}$ by $9 \mathrm{~s}$. Q $\left(P_{\text {fusion }} / P_{\text {aux }}\right)$ is greater than 10 from 8.5 (SOB) s to 27 $\mathrm{s}$, which is 37 energy confinement times. The helium density remains constant from 14 to $27 \mathrm{~s}$, which is 5 effective particle confinement times. The helium ash reaches $3 \%$ of the electron density, with $Z_{\text {eff }}$ becoming 1.4. The plasma internal inductance drops to 0.85 as the bootstrap current develops (reaching 1.6 MA) and the higher edge temperature associated with the H-mode allows more current to flow at the plasma edge. The poloidal and toroidal $\beta$ values rise to 1.2 and $3 \%$, respectively, corresponding to 
$\beta_{N}=2.42$. About $2.5 \mathrm{~V}-\mathrm{s}$ are consumed from SOF to EOB. The ratio of the line-averaged electron density to the Greenwald density reaches 0.6 .

The burn termination and plasma current rampdown phase extends from 27 to 34(EOD) s. The burn termination is initiated by reducing the plasma density, more specifically the fueling is stopped. The alpha power rapidly drops and the plasma transitions from $\mathrm{H}$ to L-mode. The auxiliary power is decreased more slowly to 15-10-5 MW to avoid high density disruptions. The plasma current is reduced during the burn termination phase, 27 to 30 (EOC) s, to $5.0 \mathrm{MA}$ to avoid flux consumption as the plasma cools, and then is reduced more quickly to $100 \mathrm{kA}$ from 30 to $34 \mathrm{~s}$. During the burn termination phase the plasma remains diverted since auxiliary heating is being applied, but then becomes limited on the inboard wall and is reduced in size during the current rampdown. The current rampdown time is chosen to avoid large negative currents near the plasma edge which can lead to disruption and vertical instability as $l_{i}$ increases rapidly. Poloidal flux is returned to the transformer throughout burn termination and current rampdown.

A series of variations on the reference discharge scenario were done to begin to address the device's flexibility. Some examples include perturbing the auxiliary power, plasma fueling, and plasma pumping. These cases showed that the $21 \mathrm{~s}$ pulse length was sufficient to examine the burn response and burn control measures. In addition, degradations of some of the most critical plasma parameter assumptions including, density peakedness, effective particle confinement time, impurity fractions, and sawtooth behavior, are examined to determine the accessible plasma operating space, and how auxiliary power, density, $\beta$, and energy confinement time can affect it.

Discharge scenarios were also developed for the high field (12.0 T and 7.7 MA) discharge, and the long pulse DD (4.0 T and 2.0 MA) scenarios. Both scenarios were shown to remain within PF coil allowables. For the high field case the plasma consumes $39.3 \mathrm{~V}$-s throughout the discharge, which brings the coil stress close to its allowable. As would be expected, this scenario has little margin for plasma parameter uncertainties. The pulse length is $12 \mathrm{~s}, \beta$ reaches $2.1 \%$, the ratio of electron to Greenwald density is 0.43 , and the $\mathrm{Q}$ value is 10.0 . The long pulse $\mathrm{DD}$ case is intended to examine non-inductive current drive without a burning plasma. It assumes $14 \mathrm{MW}$ of ICRF/FW and $6 \mathrm{MW}$ of LHCD. For the electron density of $1.2 \times 10^{20} / \mathrm{m}^{3}$ and peak temperature of $15 \mathrm{keV}, 300 \mathrm{kA}$ is driven by the FWCD and $300 \mathrm{kA}$ is driven by the LHCD. The bootstrap current provides $775 \mathrm{kA}$, leaving $625 \mathrm{kA}$ to be driven by the transformer. This case assumes H-mode operation, and has an energy confinement time of $0.2 \mathrm{~s}$. If the energy confinement is allowed to increase to 3.5 times L-mode, $100 \%$ of the current can be driven non-inductively, keeping other quantities fixed. The long pulse scenario demonstrates the flexibility in the TF coil pulse length as the TF field is reduced, and can examine the long pulse (advanced tokamak) physics issues identified by the TPX[4] project.

The examination of advanced tokamak scenarios with DT burning is in a preliminary stage. The toroidal field and plasma current would be reduced to provide a pulse length sufficient to examine the current diffusion time scale. Several issues must be addressed including auxiliary power for simultaneous heating and current drive, alpha particle losses, MHD stability and high bootstrap fractions, and the discharge trajectory to produce the equilibria.

\section{VERTICAL STABILITY AND CONTROL}

The vertical stabiliy was analized to design a passive structure capable of slowing down the plasma instability over a range of plasma parameters using Corsica[3]. Low pressure plasmas are the most unstable, so that $\beta_{p}$ was set at 0.1 (typical of preheating plasmas) for the stability calculations, and the current profile, li, was varied. A series of copper stabilizer plate geometries were examined attempting to provide a stability factor of 1.2 or greater, and incorporating the restrictions of midplane ports, slanted ports, and divertor. The stability factor is defined as $f_{s}=\left(1+\tau_{g} / \tau_{L / R}\right)$, where $\tau_{g}$ is the vertical instability 
growth time, $\tau_{L / R}$ is the longest up-down asymmetric time constant of the passive structures, and a value of $f_{s}=1$ indicates that the plasma is uncontrollable. The stability factors varied from 1.3 to 1.13 over the $l_{i}$ range of 0.7 to 1.1. The growth times corresponding to these went from 43.0 to $18.5 \mathrm{~ms}$. Although $f_{s}$ dropped below 1.2 at the higher $l_{i}$ values, this was considered satisfactory since control simulations indicated that these plasmas could be controlled without excessive power. The final passive stabilizer is composed of both inboard and outboard plates, shown in Fig. 1. The outboard plate is broken once in the toroidal direction with saddle connections between the upper and lower plates, while the inboard plate is made toroidally continuous. The plates are $1.5 \mathrm{~cm}$ thick copper. The longest up-down asymmetric L/R time constant for the entire structure is $140 \mathrm{~ms}$. The toroidal resistance of the double walled vacuum vessel is $48 \mu \Omega$, for the inboard passive plate is $4.8 \mu \Omega$, and for the outboard passive plate is about 100 $\mu \Omega$ (determined by the current path through the vacuum vessel).

Using the most unstable plasmas at high li, feedback control simulations were done with TSC. The control coils were located on the outboard side, inside the TF coil and outer vacuum vessel, but outside the inner vacuum vessel, shown in Fig. 1. Step response simulations were done to determine gains and minimum voltage limits to provide low power and satisfactory response. Random disturbance simulations are then used to determine likely current and voltage requirements during a discharge. For a $\Delta Z_{R M S}$ of $1.0 \mathrm{~cm}$ and step $\Delta Z$ of $2.0 \mathrm{~cm}$, the peak power requirements fall in the range of 5 to $10 \mathrm{MVA}$. The maximum coil voltage is $75 \mathrm{~V} / \mathrm{turn}$, with the required peak voltage falling between 50 and $75 \mathrm{~V} / \mathrm{turn}$. The peak coil currents associated with these voltages range from 55 to $75 \mathrm{kA}$-turn.

\section{CONCLUSIONS}

The PF coil system designed for FIRE has been shown to provide the reference scenario with sufficient margins to all coil allowables to compensate for plasma uncertainties. In addition, these margins allow for a high field scenario, and a long pulse DD scenario. The full discharge simulations have shown that FIRE can provide sufficient fusion power to obtain $\mathrm{Q}=10$, and can enter and remain in the H-mode. These simulations have been used in conjunction with coil heating and stress analysis, and power supply analysis to generate optimized scenarios. The simulations also provide a means to examine plasma responses and burn control, as well as, sensitivity to several plasma parameter assumptions. The vertical stability analysis shows that the passive stabilizer design in combination with the internal feedback control coils, is sufficient to provide vertical position control with reasonable power.

Work supported by U.S. DOE Contract DE-AC02-76-CHO3073

\section{References}

[1] S. C. Jardin, N. Pomphrey, and J. Delucia, "Dynamic Modeling of Tranport and Position Control of Tokamaks", J. Comput. Phys., vol. 66, p. 481, 1986.

[2] G. R. Harris, "Comparisons of Different Bootstrap Current Expressions", Rep. EUR-CEA-FC-1436, Centre d'etudes de Cadarache, Saint-Paul-lez-Durance, France, 1991.

[3] S. W. Haney, L. D. Pearlstein, R. H. Bulmer, and J. P. Freidberg, "Vertical Stability Analysis of Tokamaks Using a Variational Procedure", Plasma Physics Reports, vol. 23, p. 789, 1997.

[4] R. J. Goldston, "Physics of the Steady-State Advanced Tokamak", Phys. Plasmas, vol. 3, p. 1794, 1996. 
Table 1: PF Coil Locations

\begin{tabular}{cccccc}
\hline & $\mathrm{R}(\mathrm{m})$ & $\mathrm{Z}(\mathrm{m})$ & $\Delta \mathrm{R}(\mathrm{m})$ & $\Delta \mathrm{Z}(\mathrm{m})$ & $\mathrm{f}_{O H}$ \\
\hline CS1 & 0.610 & 0.400 & 0.400 & 0.800 & 0.3334 \\
CS2 & 0.610 & 1.025 & 0.400 & 0.400 & 0.2082 \\
$\mathrm{CS} 3$ & 0.610 & 1.475 & 0.400 & 0.400 & 0.1883 \\
PF1 & 0.786 & 1.975 & 0.325 & 0.380 & 0.1425 \\
PF2 & 1.211 & 2.211 & 0.325 & 0.380 & 0.0847 \\
PF3 & 3.050 & 2.750 & 0.400 & 0.300 & 0.0401 \\
PF4 & 4.400 & 1.000 & 0.400 & 0.300 & 0.0028 \\
\hline
\end{tabular}

Table 2: Nominal PF Coil Currents (MA) for Reference 10.0 T Discharge and Maximum Current for $\left(l_{i}, \psi_{e x t}\right)$ Variation

\begin{tabular}{lcccccccccc}
\hline & $\begin{array}{c}\mathrm{IM} \\
\mathrm{t}=0^{-}\end{array}$ & $\begin{array}{c}\mathrm{SOD} \\
\mathrm{t}=0^{+}\end{array}$ & $\begin{array}{c}\mathrm{SOF} \\
\mathrm{t}=6.0 \mathrm{~s}\end{array}$ & $\begin{array}{c}\mathrm{SOB} \\
\mathrm{t}=8.5 \mathrm{~s}\end{array}$ & $\begin{array}{c}\text { EOB } \\
\mathrm{t}=27.0 \mathrm{~s}\end{array}$ & $\begin{array}{c}\text { EOC } \\
\mathrm{t}=30.0 \mathrm{~s}\end{array}$ & $\begin{array}{c}\text { EOD } \\
\mathrm{t}=34.0 \mathrm{~s}\end{array}$ & $\begin{array}{c}\text { SOF } \\
I_{\max }\end{array}$ & $\begin{array}{c}\text { SOB } \\
I_{\max }\end{array}$ & $\begin{array}{c}\text { EOB } \\
I_{\max }\end{array}$ \\
\hline CS1 & 8.51 & 7.46 & -12.0 & -10.7 & -11.7 & -9.90 & 0.59 & -14.5 & -13.0 & -14.1 \\
CS2 & 5.97 & 5.23 & 1.50 & 1.55 & 0.858 & 2.72 & 0.38 & 3.18 & 2.80 & -3.12 \\
CS3 & 3.03 & 2.66 & 1.72 & 1.51 & 0.96 & 2.69 & 0.22 & 5.56 & 4.81 & 4.12 \\
PF1 & 2.38 & 2.08 & 4.75 & 4.05 & 3.70 & 2.70 & 0.15 & 5.60 & 4.50 & 4.55 \\
PF2 & 3.86 & 3.37 & 4.75 & 4.05 & 3.70 & 2.70 & 0.18 & 5.60 & 4.50 & 4.55 \\
PF3 & 0.45 & 0.39 & -3.36 & -1.48 & -1.58 & -1.19 & 0.00 & -4.72 & -2.99 & -3.06 \\
PF4 & 0.17 & 0.15 & -2.81 & -3.95 & -3.96 & -2.61 & 0.02 & -3.60 & -4.63 & 4.65 \\
$I_{p}$ & 0.00 & 0.10 & 6.44 & 6.44 & 6.44 & 5.00 & 0.10 & 6.44 & 6.44 & 6.44 \\
$\psi_{e x t}$ & -16.0 & -14.0 & 17.6 & 18.1 & 20.1 & 12.8 & -0.90 & $-5 /+5$ & $-5 /+5$ & $-5 /+5$ \\
$\beta_{p}$ & 0.00 & 0.25 & 0.40 & 1.20 & 1.20 & 0.14 & 0.29 & 0.40 & 1.20 & 1.20 \\
$l_{i}$ & 0.00 & 1.67 & 0.85 & 0.85 & 0.85 & 1.00 & 1.30 & $-.1 /+.15$ & $-.1 /+.15$ & $-.1 /+.15$ \\
\hline
\end{tabular}




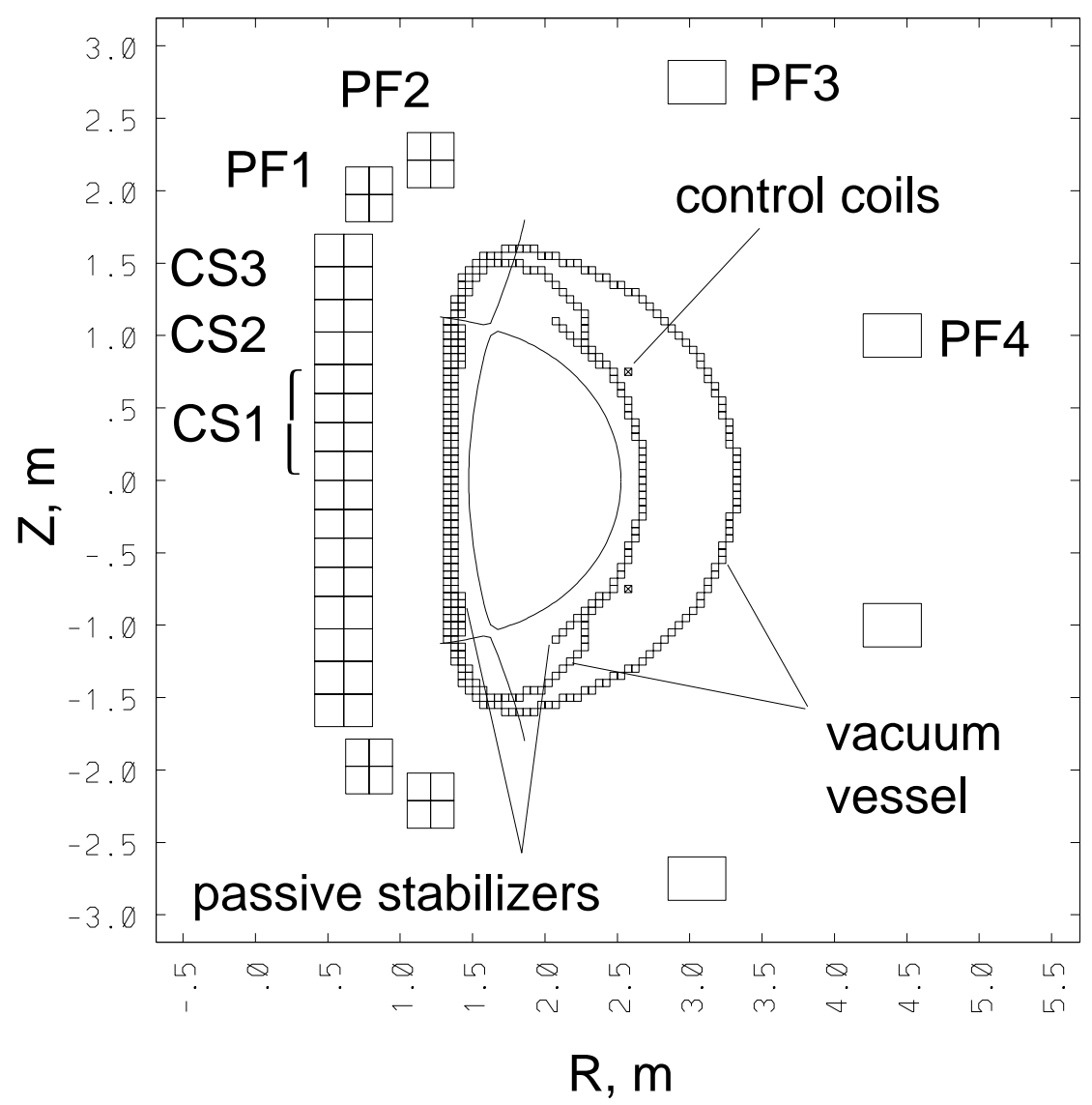

Figure 1: Model of plasma, structures, and PF coils used in equilibrium and dynamic discharge calculations 


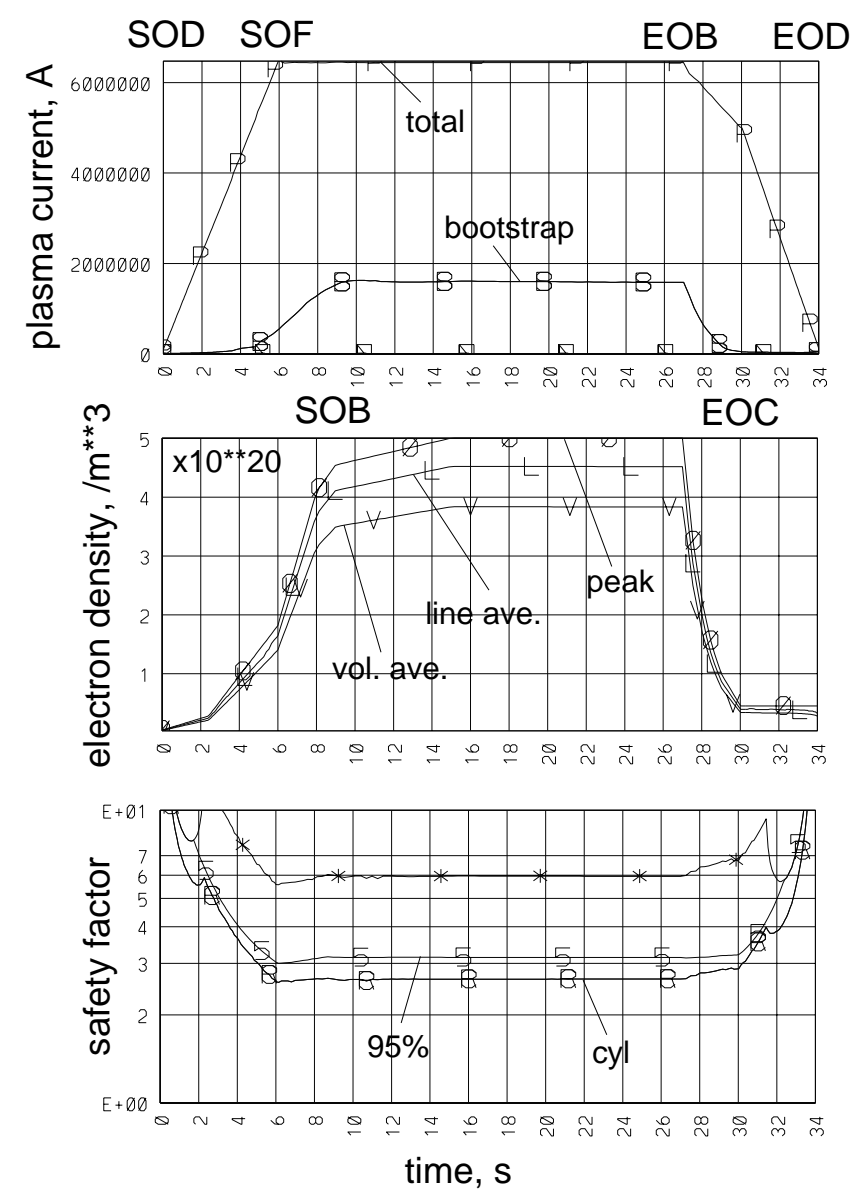

Figure 2: Time histories of plasma current, electron density, and safety factor for the reference $10 \mathrm{~T}$ and 6.44 MA discharge 


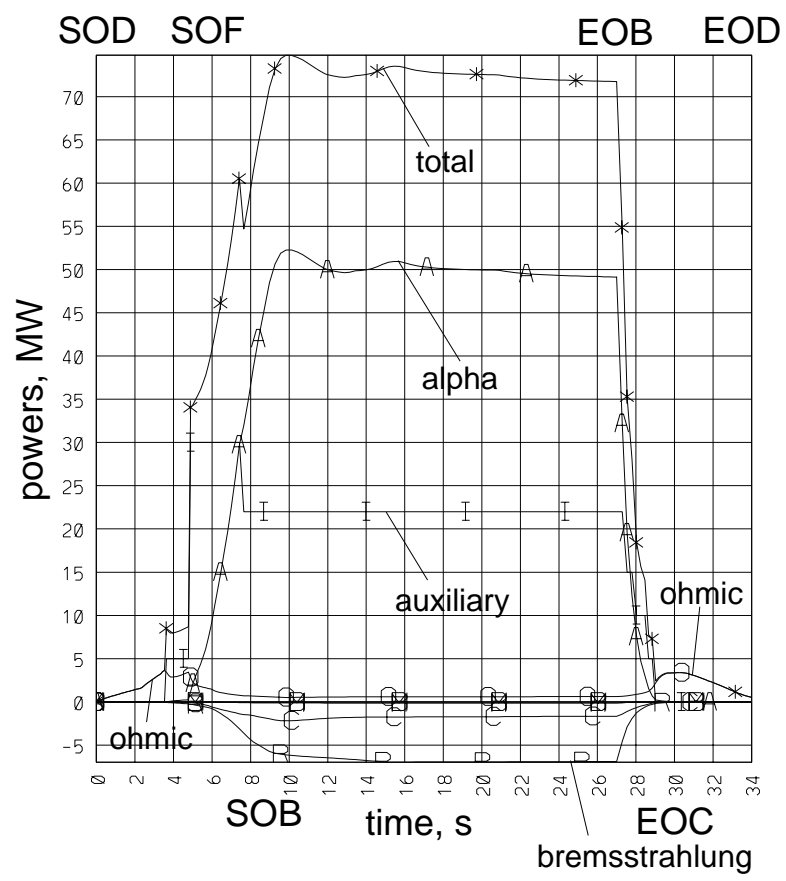

Figure 3: Time histories of the various plasma powers for the reference $10 \mathrm{~T}$ and $6.44 \mathrm{MA}$ discharge 